\title{
NASA GRC UAS Project \\ Communications Modeling and Simulation Status
}

\author{
Presenter - Greg Kubat, Systems Engineer \\ Vantage Partners, LLC. \\ 3000 Aerospace Parkway \\ Brookpark, Ohio 44142
}

Modeling and Simulation Team Contributors

Steve Bretmersky / Justin Dailey - MTI Systems

Goutam Satapathy / David Ditzenberger / Chris Ye / David Thorpe - Intelligent Automation Inc.

Christine Chevalier / Thanh Nguyen - Vantage Partners, LLC.

Rafael Apaza (Lead) - NASA GRC

2013 Integrated Communication, Navigation, and Surveillance (ICNS) Conference 23-25 April 2013

Herndon, Virginia 


\section{GRC UAS Project - Communications Modeling and Simulation}

\section{GRC UAS Communications Modeling - Simulation Objectives}

Primary Objective of Simulation Activity

Use Modeling and Simulation to perform analysis to support recommendations for integration of UAS CNPC systems for safe and efficient operation of UAS in the NAS.

Develop Models of representative Radio Technologies for UAS CNPC operations

- Investigate UAS Radio Technologies Design and Performance for UAS CNPC

- Assist with GRC UAS Flight Test Radio development

- Generate recommendations for UAS CNPC radio requirements (from simulation results)

Develop Large-scale , NAS-wide Simulation capability with NAS ATM Communications and integrated UAS CNPC Communications

- Perform Simulations with varied UAS in the NAS Communication System Architectures

- Analyze NAS ATM Communication System Performance with the introduction of UAS ATM communications traffic.

- Analyze the Performance Impact on NAS ATM Communication System operations due to UAS Air Traffic loading

- Provide recommendations for UAS in the NAS Comm System Architecture requirements 


\section{GRC UAS Project - Communications Modeling and Simulation}

\section{GRC UAS Communications Mod/Sim Approach}

\section{Develop UAS Radio Models using Opnet Modeler}

Opnet provides Discrete event modeling environment for simulation of Processes, Protocols,

Applications, System Devices and Communications Devices

- Develop Models with varied radio technologies (for Characterization/Perf. Comparisons)

- Develop One Model to match the ongoing GRC Flight Test Radio design. (Validated Model)

- Perform simulations within the Opnet environment to study detailed Radio performance

\section{Develop Large-Scale UAS in the NAS Comm Architectures using NASA 'ACES' application}

ACES = Airspace Concepts Evaluation System (Developed at NASA ARC for Airspace Concept Research)

- Provides NAS fast-time, airspace simulations with NAS ATM Component Models

- Customizable, Single-flight to Day-in-the-NAS flight traffic loading

- Aircraft Models for Commercial and General Aviation A/C and several UAS (recently developed)

- Use of ACES gives our simulations complete NAS Airspace Architecture and Operations

- Leverage previous experience integrating Comm Models/Comm Infrastructure into ACES

- Develop one Relay and one Non-Relay UAS Comm System Architecture for analysis.

\section{Integrate Opnet CNPC Radio models into Large-scale Simulation Architectures}

- Provides interoperability between NAS Airspace operations, NAS air traffic loads, UAS Air traffic loads, existing ACES ATM Comm and new CNPC UAS Comm Systems

- Allows us to characterize NAS Comm Message Traffic levels and profiles that will exist with UAS

- Provides data to assess NAS ATM Comm Performance/Impacts and UAS CNPC link performance in same simulation environment. 


\section{GRC UAS Project - Communications Modeling and Simulation}

\section{Technical Assessments}

Performed a Comm Technology Assessment to determine best suited Radio Technologies for a CNPC Datalink Radio

- Evaluated over 40 technologies using technology rating methodology

- Selected 802.16, LTE, P-34, TETRA Release 2 (TEDS) as top four candidate technologies

- 802.16 will be used for GRC Flight Test Radio Design

- LTE, P-34, TETRA Release 2 (TEDS) radios will be developed for performance comparison

Performed Architecture Assessment of RTCA SC203 (CC005) Communication Architectures

- Evaluated 8 High Level Architecture Concepts (4 Relay / 4 Non-relay) presented in CC005

- Interpreted the Architecture Options (based on authors definitions for link functionality) and developed next lower level system drawings.

- Incorporated Datalink radio as Primary CNPC link in parallel with Satcom

- Performed a functional evaluation to determine the ability of each architecture to provide connectivity required for critical UAS operating scenarios

- Performed a technically subjective evaluation of subsystems using a rating/ranking process. (Eval Criteria: Complexity, Technical Readiness, Scalability, Flexibility)

- Resulted in the selection of CCO05 UA Relay \#4 and UA Non-Relay \#4 as our target Architectures. 


\section{GRC UAS Project - Communications Modeling and Simulation}

\section{Opnet Radio Model - CNPC Radio Model Development Approach}

RADIO MODEL INFRASTRUCTURE
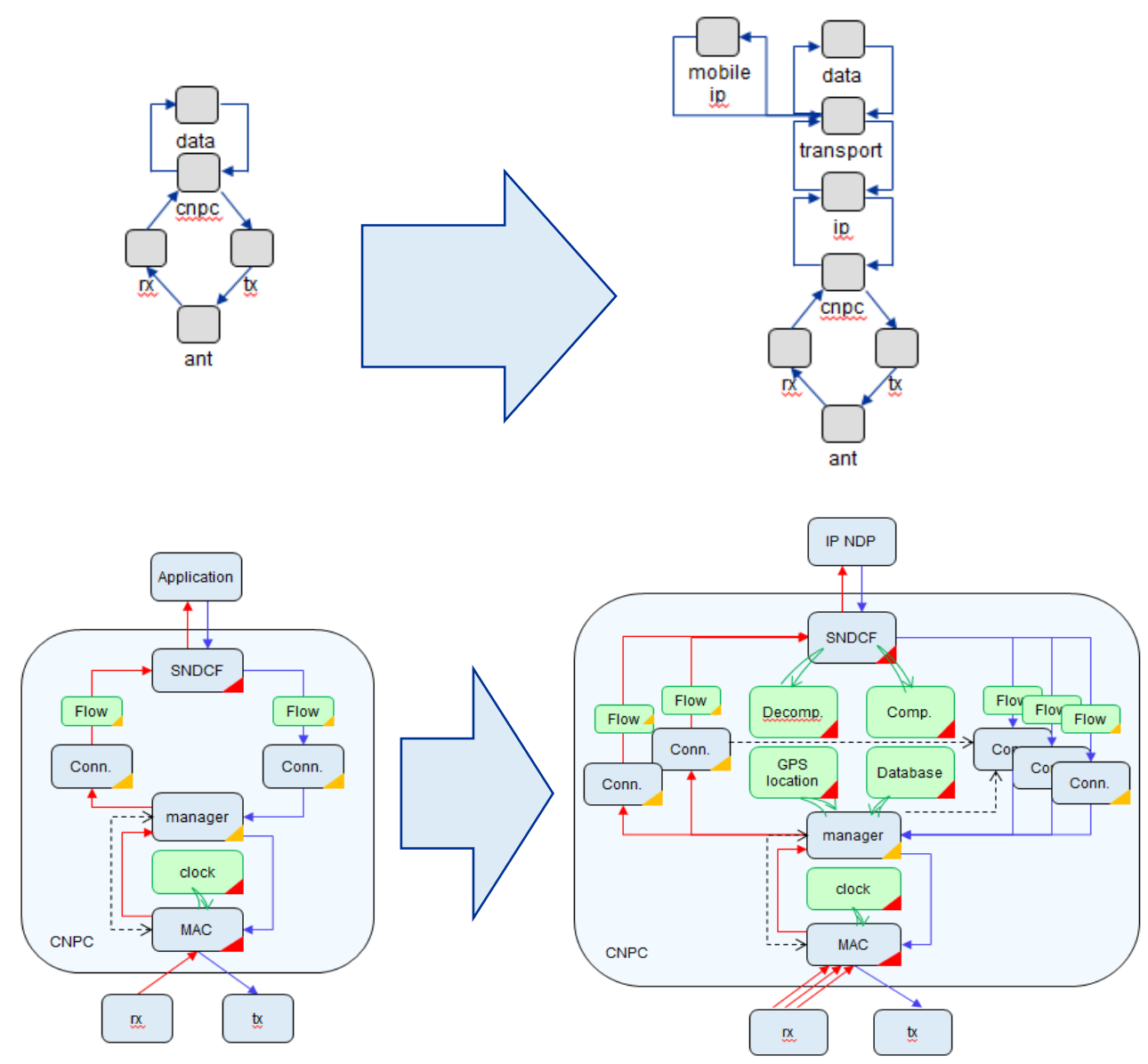

First generation
Second generation
- Develop the CNPC radio model architecture to follow the spiral development approach being used for GRC Flight Test radios

- Start simple, add features and functionality in second and third spiral

- Increase functionality / increase analysis detail

- Develop a platform compatible with the prototype radio as well as the alternative technologies 
GRC UAS Project - Communications Modeling and Simulation

Opnet Radio Model - High Level Radio Model Elements

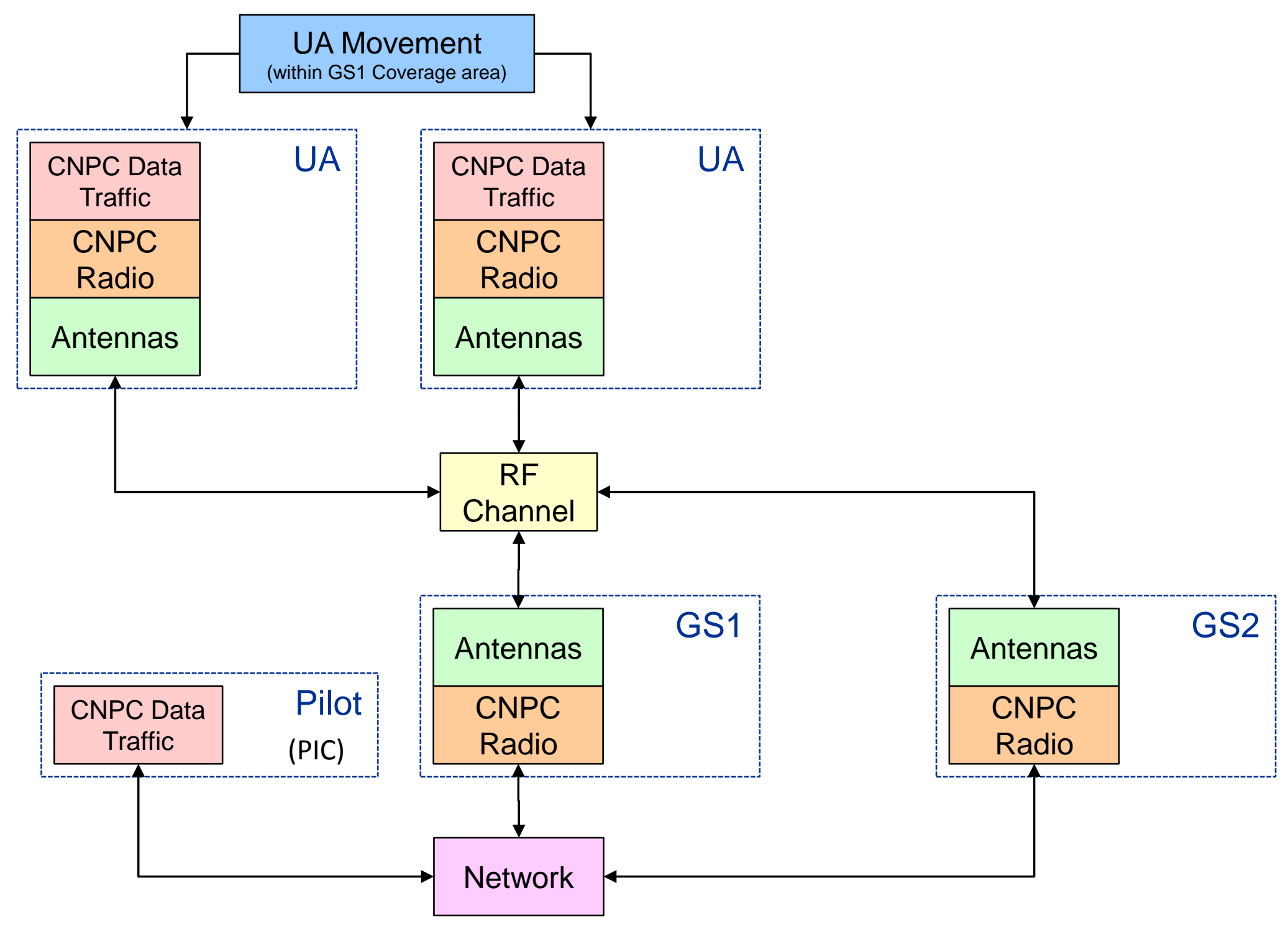




\section{GRC UAS Project - Communications Modeling and Simulation}

Opnet Radio Model - CNPC Radio Overview

- Uplink: Time Division Multiple Access

- Downlink: Frequency Division Multiple Access

- Uplink/Downlink Duplexing: Time Division Duplexing

- L and C Band radios platforms
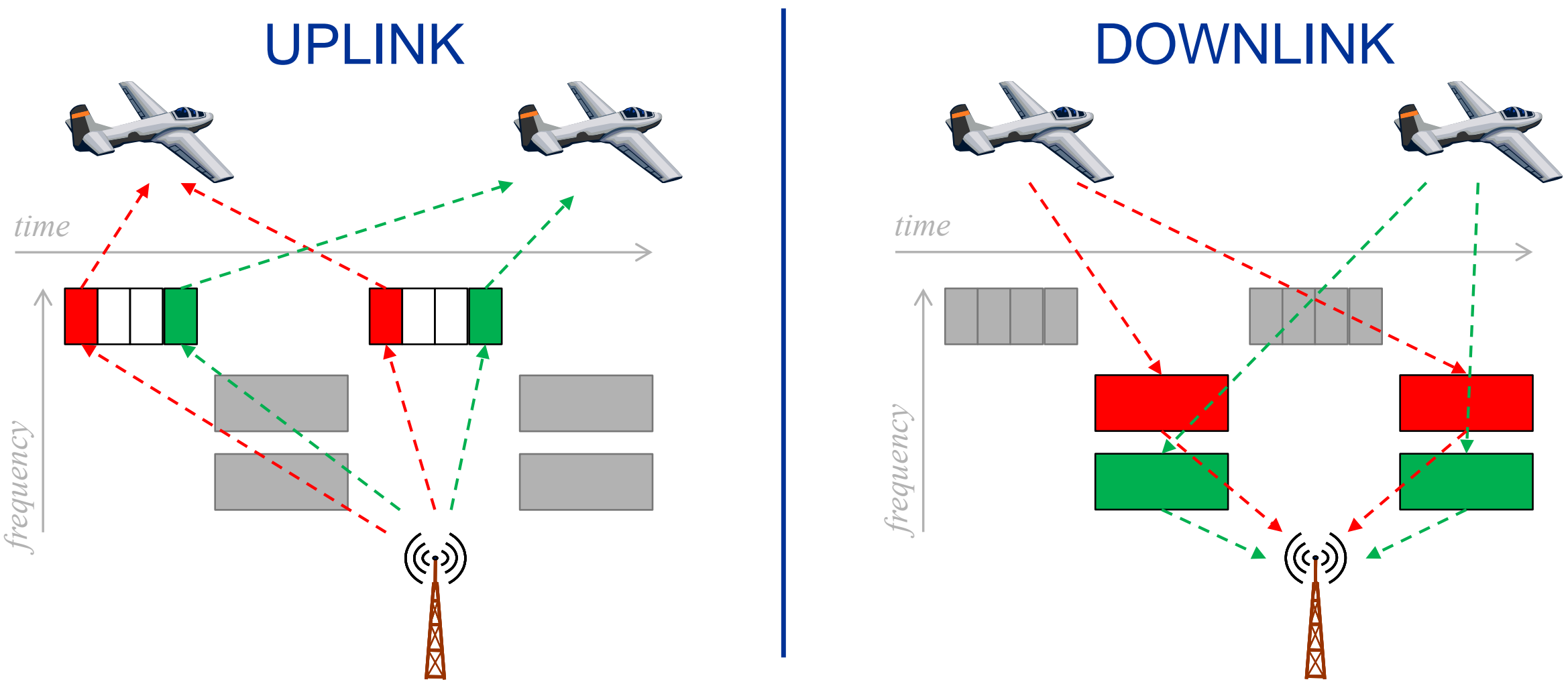
GRC UAS Project - Communications Modeling and Simulation

Large Scale - UA Relay 4 Architecture - Functional Diagram/Capabilities

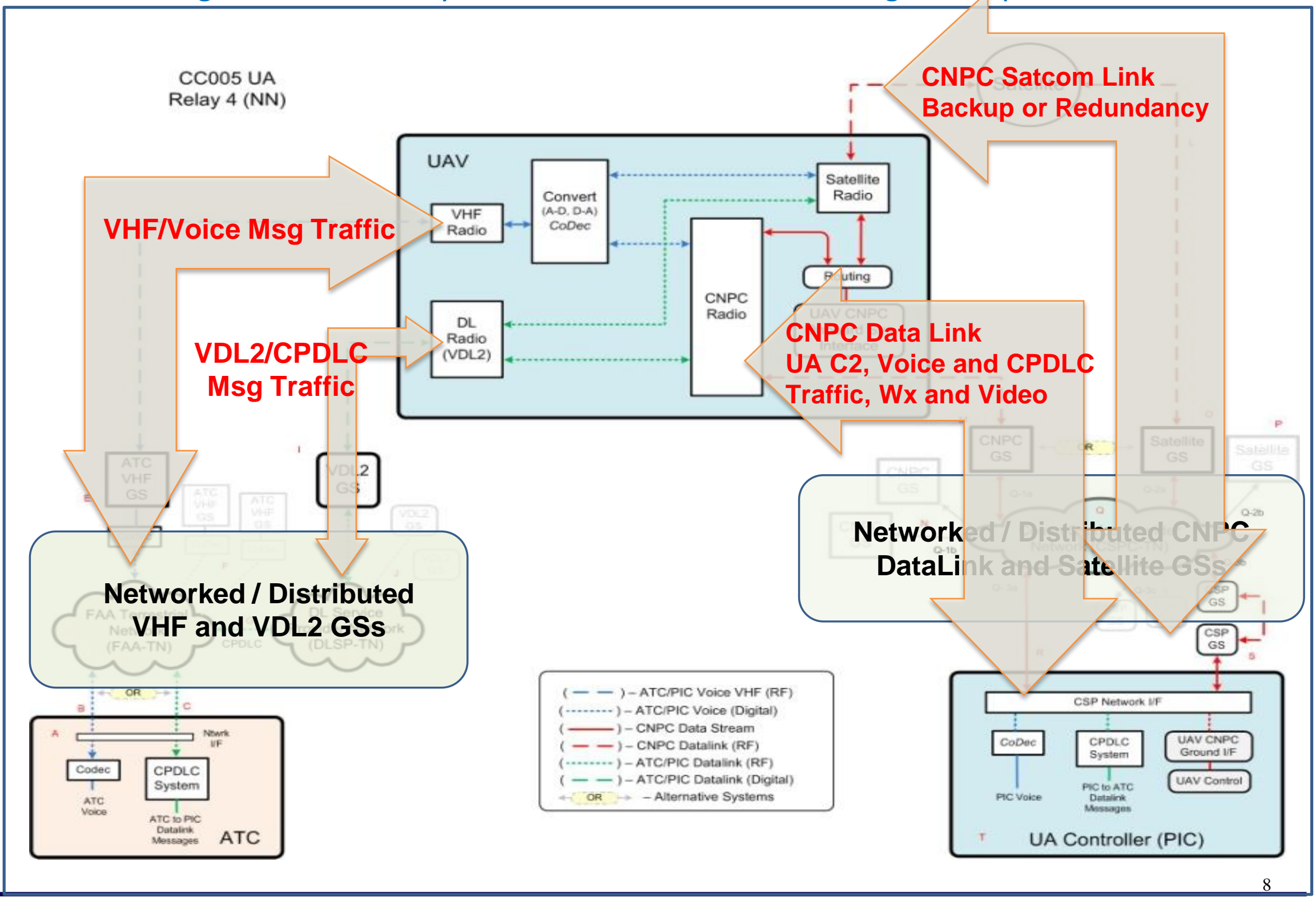


Large Scale - UA Non-Relay 4 Architecture - Functional Diagram/Capabilities

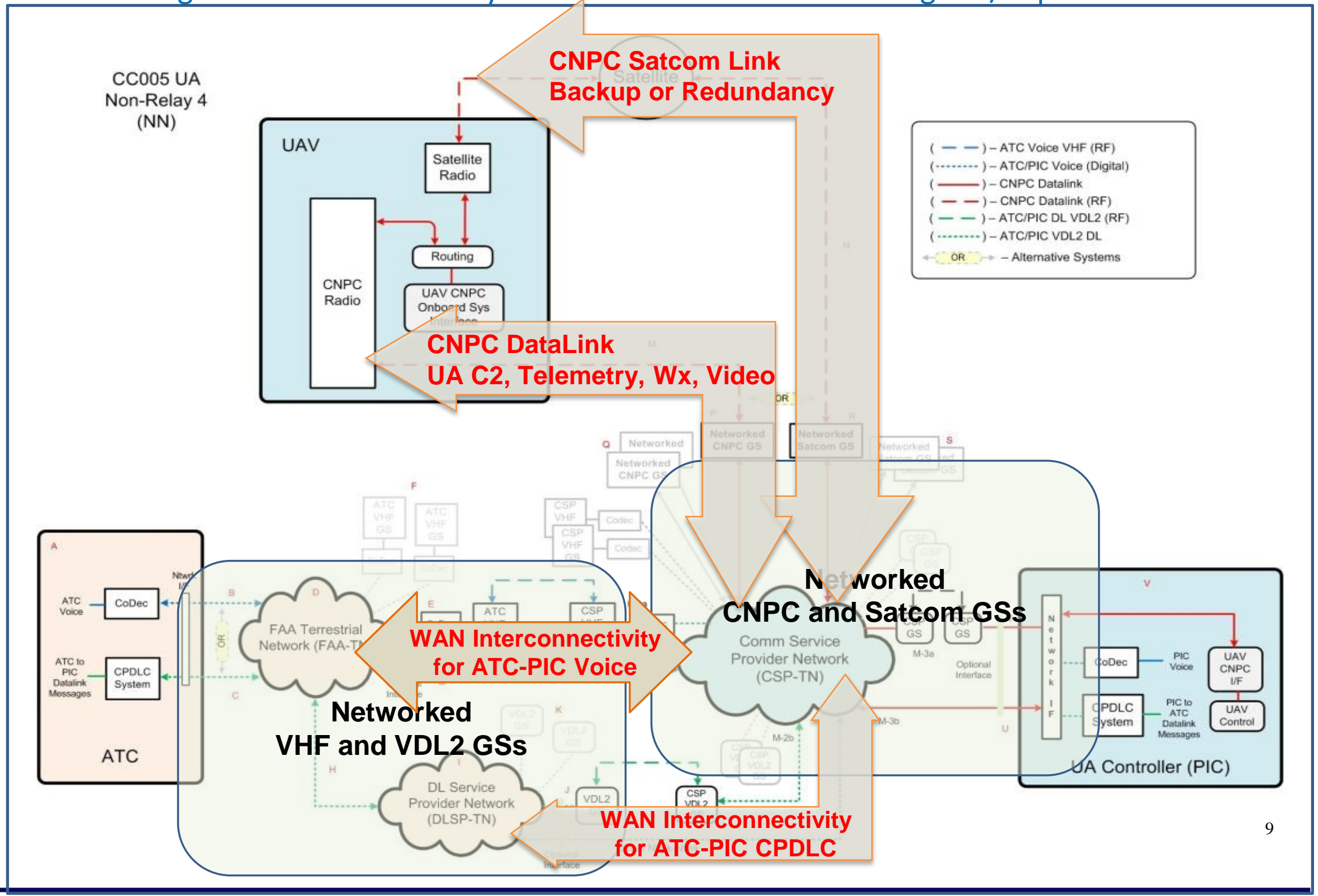


UA Relay - Large-scale (ACES-Opnet) Simulation Architecture

Large-scale UAS Comm Architecture (Relay Operation)

\section{Opnet Modeler - UA CNPC Link Radios and GS Models (Run independently in Opnet)}

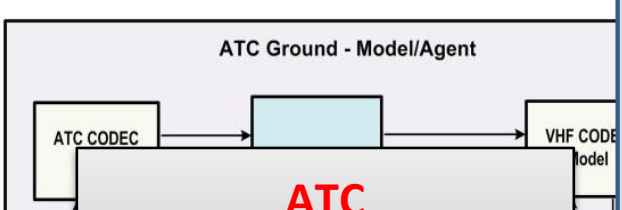
System Components

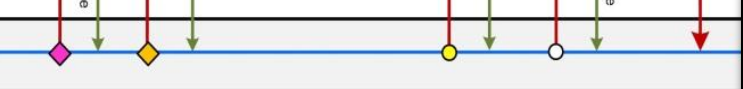

L Piloted A/C VHF
Radio

VHF

Comm
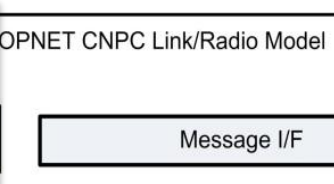

Message I/F

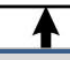

Models

for UAS

and

Piloted

$\mathrm{A} / \mathrm{C}$

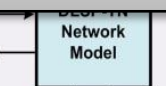
Comm Models for UAS and Piloted $\mathrm{A} / \mathrm{C}$ A/C

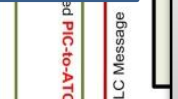

VHF/VDL2 Comm Message I/F to Opnet CNPC
ATC Ground - Model/Agen

Network/Ground
Opnet Modeler

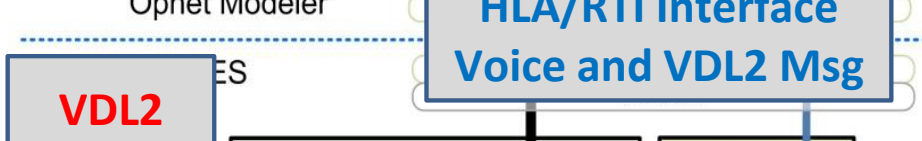

$\uparrow$ Radio Model (

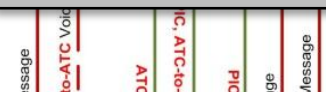
PIC/Pilot to ATC Message Flow

\section{ACES - Simulation Environment}

Opnet (ACES KTG)

Trajectory Generator

(UA flights only - also running in ACES) ACES

PIC Ground - Model/Agent

$$
\text { PIC }
$$

Network/Ground System Components

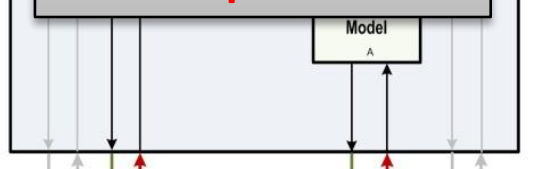<smiles>C1CC2CCC12</smiles>
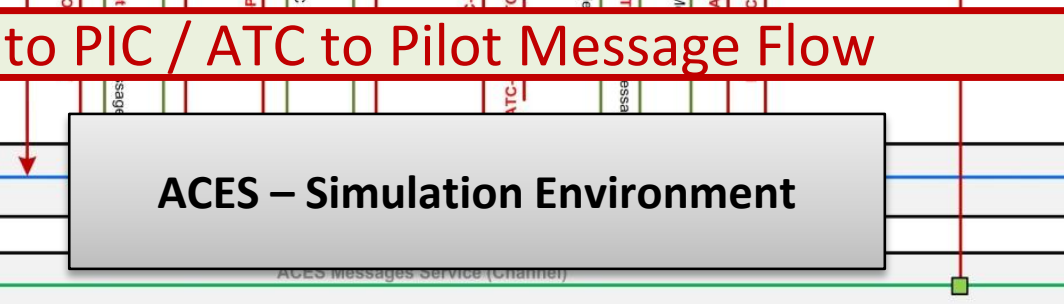


\section{GRC UAS Project - Communications Modeling/Simulation}

UA Non-relay Large-scale (ACES-Opnet) Simulation Architecture

Large-scale UAS Comm Architecture

(Non-Relay Operation)

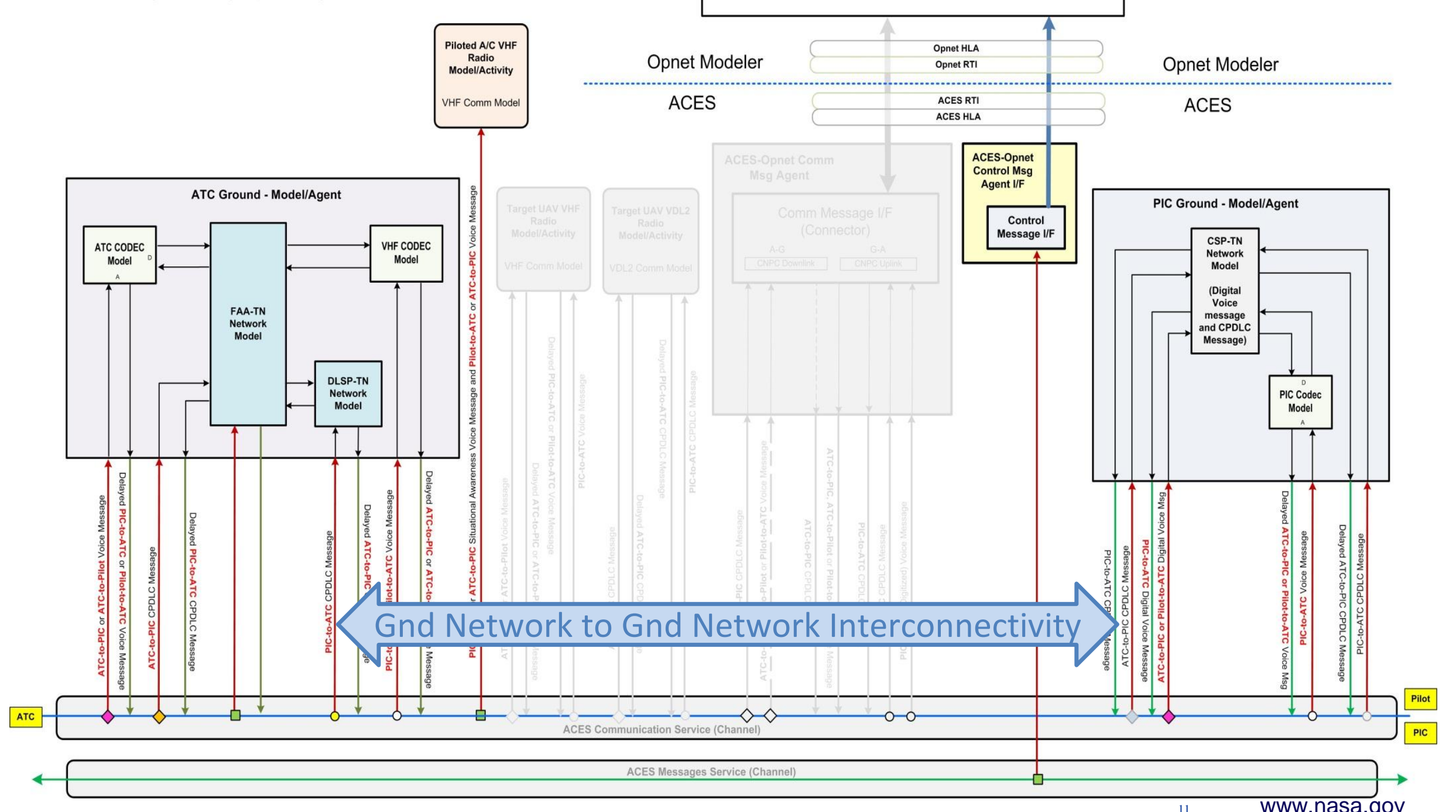




\section{GRC UAS Project - Communications Modeling/Simulation}

\section{Opnet Radio Models - Development Status}

Completed Radio Technologies down-select for GRC Flight Test Radio and 3 other optional technologies

$>$ Defined model requirements and assumptions

$>$ Defined the radio model architecture for all radio models (Common architecture/framework)

Completed first Prototype Radio Model (Gen1 - for GRC FT Radio) in Dec 2012 - basic model framework and operation.

$>$ Developed the framework and test plan for verification and validation

$>$ Performed Verification of Gen1 Radio Model

$>$ Preparing for validation against Gen1 prototype radio

- Currently working with the Datalink team on data loading and test designs

$>$ Preparing for Gen2 prototype development (Enhanced version of initial prototype) 
GRC UAS Project - Communications Modeling/Simulation Large-Scale Simulation - Development Status

Completed Down-select of Relay and Non-Relay Architectures

Completed Architecture Concept Design for ACES LS Simulation (Relay and Non-relay)

Completed Architecture Models development and testing (Relay and Non-relay)

Currently adding Msg failure/Msg sender Wait-time conditions to Voice Model in the Relay/Nonrelay architectures.

Currently testing the final integration of the ACES KTG in Opnet Modeler

- Transfering Aircraft State data to Opnet Modeler (for multi Aircraft Simulations)

Verified HLA/RTI message transfers and timing synchronization between ACES and Opnet

In process of developing the ACES to Opnet Comm Message Interface operations

- Defining the ACES-Opnet RTI Fed file for transferring Voice/CPDLC Msg data (Relay Architecture)

Completed initial/baseline Voice and CPDLC Message definitions and integration into ACES

Currently defining ACES to Opnet Control messages (Aircraft or airspace info that will impact Opnet radio operation)

(e.g. UAV airspace transitions - vary CNPC link data rates)

LS Simulation Startup procedure for ACES/Opnet/KTG Initialization - 70\% Complete 